\title{
Evaluation and diversity analysis in Indian mustard [Brassica juncea (L.) Czern \& Coss.] germplasm accessions on the basis of principal component analysis
}

\author{
Ram Avtar*, Manmohan, Minakshi Jattan, Babita Rani, Nisha Kumari, N. K. Thakral \\ and R. K. Sheoran \\ Department of Genetics and Plant Breeding, C.C.S. Haryana Agricultural University, Hisar -125004 (Haryana), \\ INDIA \\ *Corresponding author. E-mail: ramavtarola@yahoo.in
}

Received: December 7, 2016; Revised received: August 10, 2017; Accepted: November 8, 2017

\begin{abstract}
Principal component analysis was carried out with 20 morphological traits (including quantitative as well as qualitative) among 96 germplasm lines of Indian mustard [Brassica juncea (L.) Czern \& Coss.]. Principal factor analysis led to the identification of eight principal components (PCs) which explained about $70.41 \%$ variability. The first principal component (PC1) explained $16.21 \%$ of the total variation. The remaining PC's explained progressively lesser and lesser of the total variation. Varimax Rotation enabled loading of similar type of variables on a common principal factor (PF) permitting to designate them as yield factor, maturity factor and oil factor etc. Based on PF scores and cluster mean values the germplasm accessions viz., RC2, RC32 and RC51 (cluster I), RC95 and RC96 (cluster X) were found superior for seed yield/plant and yield related factors like primary and secondary branches/plant; while the accessions RC34, RC185 and RC195 (cluster III) and RC53 (cluster VIII) were found superior for oil content. These accessions may further be utilized in breeding programmes for evolving mustard varieties having high seed yield and oil content. Hierarchical cluster analysis resulted into ten clusters containing two to 26 accessions. The results of cluster and principal factor analyses were in confirmation of each other.
\end{abstract}

Keywords: Cluster, Diversity, Germplasm, Indian mustard, Principal component

\section{INTRODUCTION}

B. juncea (L.) Czern \& Coss., commonly known as Indian mustard is an amphidiploid species that originated through the interspecific hybridization of Brassica rapa and Brassica nigra (Nagaharu U, 1935). In most of the regions of the world, its cultivation has increased drastically during the last decades due to its inherent high yielding ability and relative tolerance to biotic and abiotic stresses and, by now; it is the third largest contributor of the world supply of vegetable oil. It is one of the most important oil and protein rich annual crops in the world. At national level it is grown over an area of 6.45 million ha with production and productivity of 7.28 million tons and $1128 \mathrm{~kg} / \mathrm{ha}$, respectively (Anonymous, 2015).

The utilization of a species into any crop improvement breeding programme depends not only on the degree of genetic diversity it holds, but also on the precise information regarding genetic divergence and relatedness among breeding materials. Agronomic characterization is a useful tool for the genotypic classification, varietal identification in seed production programmes through DUS traits and it also allows plant breeders to select valuable genetic resources to be strategically utilized in different breeding programmes (Singh et al., 2013). Therefore, the choice of suitable parents is a matter of great concern to the plant breeders. The principal component and factor analysis is an important tool for the assessment of genetic divergence among the parents/ genotypes and also to assess the relative contribution of particular trait to the total variability. Principal component analysis also helps in identifying most relevant characters by explaining the total variation in the original set of variables with as few of the components as possible and reduces the complexity or dimension of the problem (Zaman et al., 2010). Thus, keeping all this in view, the present study was planned with the objectives of assessment of genetic divergence and principal component and factor analyses in 96 germplasm accessions of Indian mustard.

\section{MATERIALS AND METHODS}

Ninety six germplasm accessions (Table 1) of Indian mustard (Brassica juncea) were grown in paired rows of $5 \mathrm{~m}$ length each at a spacing of $30 \times 15 \mathrm{~cm}$ row to row and plant to plant at Oilseeds Research Area, Department of Genetics and Plant Breeding, CCS HAU, Hisar during rabi, 2015-16. Recommended package of practices to raise a good crop was followed. Observa- 
tions were recorded on five randomly selected plants in each germplasm accession on 14 morphological quantitative variables viz., plant height $(\mathrm{cm})$, days to flowering, days to maturity, number of primary branches/ plant, number of secondary branches/plant, seed yield/ plant $(\mathrm{g})$, seeds/siliqua, main shoot length $(\mathrm{cm})$, siliqua length $(\mathrm{cm})$, siliqua density, siliqua number on main shoot, number of lobes/leaf, 1000-seed weight (g) and oil content $(\%)$.

Data were also recorded on six qualitative traits by giving scores in accordance with the standard DUS descriptor. These traits were leaf angle [1-erect $\left(>85^{\circ}\right)$, 3 -semi-erect $\left(66-85^{0}\right)$, 5-open $\left(46-65^{0}\right)$, 7-semi prostrate $\left(31-45^{0}\right)$ and 9-prostrate $\left.\left(<31^{0}\right)\right]$, leaf hairiness $(1-$ absent, 3-sparse, 7-dense), leaf colour (1-light green, 2 -medium green, 3-dark green, 4-purple green, 5purple), leaf dentations (1-entire, 3-auriculate, 5-lyrate, 7-pointed), siliqua angle with main shoot [3-appressed $\left(<21^{0}\right)$, 5-semi-appressed $\left(21-30^{0}\right), \quad 7$-open $\left.\left(>30^{0}\right)\right]$ and seed colour (1-yellow, 2-dull grey, 3-reddish brown, 4-brown, 5-black).

Principal factor and cluster analyses were carried out using SPSS 10.0. Principal factor analysis was carried out using principal component method for factor extraction. The principal components (PCs) with eigen roots more than one were retained. As the initial factors loading were not clearly interpretable, the factor axes were rotated using Varimax Rotation. The correlation values $\geq 0.5$ between the traits and principal components were considered for constructing the relationship between the traits and that principal factor (PF). Principal factor scores were calculated using Anderson-Rubin method. Scatter plots were drawn using two main Principal Factors in order to identify the most distinct and useful accessions with desirable traits in different clusters. UPGMA (Unweighted PairGroup Method using Arithmetic Averages) method of Hierarchical Cluster analysis was utilized with city block distances to classify all the 96 germplasm accessions and dendrogram was prepared using the rescaled distances. Based on the method suggested by Romesburg (1990), the dendrogram was cut to form the clusters.

\section{RESULTS AND DISCUSSION}

Principal component analysis indicated that only the first eight principal components (PCs) showed eigen values more than one and they cumulatively explained $70.41 \%$ of the total variability. The first PC (PC1) explained $16.21 \%$ of the total variation and the remaining seven principal components explained 11.19, 9.36, $8.71,7.07,6.69,6.01$ and $5.13 \%$ variation, respectively (Table 2). The first one absorbed and accounted for maximum proportion of total variability in the set of all PCs and the remaining ones accounted for progressively lesser and lesser amount of variation. Similar results have also been reported earlier by Zada et al. (2013) in Ethiopian mustard; Avtar et al. (2014) in toria and Neeru et al. (2015) in Indian mustard.

The analysis without rotation of axes failed to load all the variables signifying that it could not offer much information regarding the idea of correlation between the variables and the principal components. Varimax Rotation was applied and this resulted in loading of all the variables on different principal components. Factors' loadings of different variables thus obtained are

Table 1. List of 96 germplasm accessions of Indian mustard.

\begin{tabular}{llllllll}
\hline Sr. No. & Accession No. & Sr. No. & Accession No. & Sr. No. & Accession No. & Sr. No. & Accession No. \\
\hline 1 & RC2 & 25 & RC34 & 49 & RC91 & 73 & RC152 \\
2 & RC5 & 26 & RC35 & 50 & RC93 & 74 & RC154 \\
3 & RC6 & 27 & RC36 & 51 & RC95 & 75 & RC161 \\
4 & RC7 & 28 & RC37 & 52 & RC96 & 76 & RC162 \\
5 & RC8 & 29 & RC38 & 53 & RC99 & 77 & RC163 \\
6 & RC12 & 30 & RC46 & 54 & RC102 & 78 & RC164 \\
7 & RC13 & 31 & RC47 & 55 & RC104 & 79 & RC165 \\
8 & RC14 & 32 & RC48 & 56 & RC105 & 80 & RC166 \\
9 & RC15 & 33 & RC49 & 57 & RC106 & 81 & RC171 \\
10 & RC18 & 34 & RC50 & 58 & RC107 & 82 & RC174 \\
11 & RC20 & 35 & RC51 & 59 & RC108 & 83 & RC175 \\
12 & RC21 & 36 & RC52 & 60 & RC110 & 84 & RC185 \\
13 & RC22 & 37 & RC53 & 61 & RC111 & 85 & RC195 \\
14 & RC23 & 38 & RC54 & 62 & RC112 & 86 & RC260 \\
15 & RC24 & 39 & RC57 & 63 & RC114 & 87 & RC261 \\
16 & RC25 & 40 & RC61 & 64 & RC116 & 88 & RC263 \\
17 & RC26 & 41 & RC74 & 65 & RC118 & 89 & RC264 \\
18 & RC27 & 42 & RC77 & 66 & RC127 & 90 & RC265 \\
19 & RC28 & 43 & RC78 & 67 & RC129 & 91 & RC268 \\
20 & RC29 & 44 & RC81 & 68 & RC134 & 92 & RC270 \\
21 & RC30 & 45 & RC85 & 69 & RC135 & 93 & RC273 \\
22 & RC31 & 46 & RC86 & 70 & RC142 & 94 & RC275 \\
23 & RC32 & 47 & RC87 & 71 & RC148 & 95 & RC280 \\
24 & RC33 & 48 & RC89 & 72 & RC150 & 96 & RC283 \\
\hline
\end{tabular}


Table 2. Total variance explained by different Principal Components among Indian mustard accessions.

\begin{tabular}{llll}
\hline $\begin{array}{l}\text { Principal } \\
\text { component }\end{array}$ & $\begin{array}{l}\text { Eigen } \\
\text { value }\end{array}$ & $\begin{array}{l}\text { Per cent } \\
\text { variance }\end{array}$ & $\begin{array}{l}\text { Per cent cumula- } \\
\text { tive variance }\end{array}$ \\
\hline 1 & 3.24 & 16.216 & 16.216 \\
2 & 2.23 & 11.193 & 27.409 \\
3 & 1.87 & 9.364 & 36.773 \\
4 & 1.74 & 8.716 & 45.488 \\
5 & 1.41 & 7.077 & 52.565 \\
6 & 1.34 & 6.699 & 59.264 \\
7 & 1.20 & 6.015 & 65.280 \\
8 & 1.02 & 5.130 & 70.410 \\
\hline
\end{tabular}

presented in Table 3. The first principal factor (PF1) ascribed for three variables in total could be designated as yield factor as it enabled high loadings of seed yield with two of its most important component traits viz. primary and secondary branches/ plant. PF2 had high loadings of three variables i.e. days to flowering, plant height and days to maturity and could be designated as maturity factor. PF3 had high loadings of two variables i.e. seeds/siliqua and main shoot length. PF4 could be called as siliqua factor as three variables viz.siliqua length, siliqua density and siliqua number on main shoot were loaded on this factor. Three variables viz. number of lobes/leaf, leaf dentations and 1000-seed weight were loaded on the principle factor 5. PF6 could be designated as oil factor as only oil content variable is loaded on this factor. Three variables viz. leaf colour, seed colour and leaf hairiness were loaded on the principle factor 7 and could be designated as colour factor. PF8 had high loadings of two variables i.e. leaf angle and siliqua angle and could be designated as angle factor. Avtar et al. (2014) in toria and Singh et al. (2014) in Indian mustard also reported loading of similar type of variables on a common principal factor (PF).

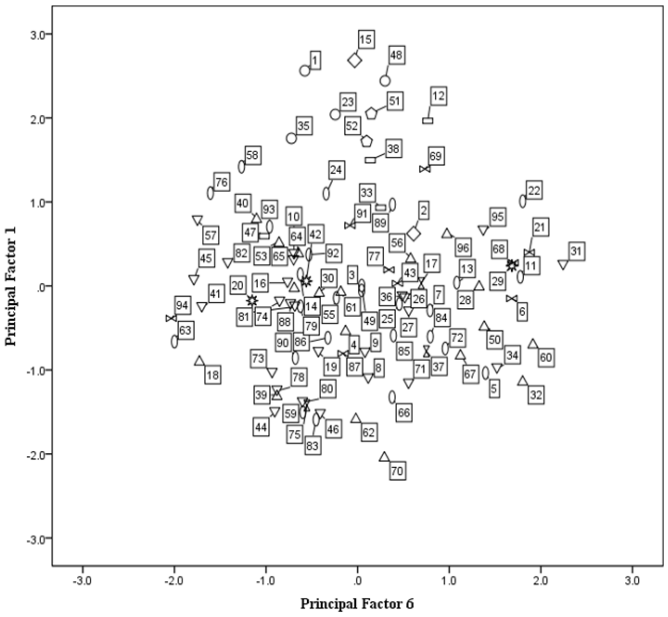

Fig. 1. Distribution of Indian mustard accessions based on Principal Factors 1 and 6.

From the present analysis it was observed that number of primary branches/plant, number of secondary branches/plant, seed yield/plant, days to flowering, plant height, days to maturity, seeds/siliqua and main shoot length were the major distinct variability contributing traits which accounted for nearly half (36.77\%) of the total variation $(70.41 \%)$ in the set of 96 germplasm accessions. Thus, the successful transformation of 20 morphological variables into eight independent principal factors by means of grouping of similar type of variables on different principal factors elaborated and explained $70.41 \%$ of the variability of the original set. These findings are in tune with those obtained by Neeru et al. (2015) in Indian mustard.

The UPGMA method with City Block distances in hierarchical cluster analysis divided the accessions into ten clusters (C). Cluster membership of different accessions is presented in Table 4. Maximum number of

Table 3. Factor loadings of different characters with respect to different Principal Factors (Varimax rotation) in Indian mustard accessions.

\begin{tabular}{lllllllll}
\hline Traits & PF 1 & PF 2 & PF 3 & PF 4 & PF 5 & PF 6 & PF 7 & PF 8 \\
\hline No. of secondary branches/plant & $0.876^{*}$ & 0.077 & -0.160 & 0.105 & -0.097 & 0.050 & 0.000 & 0.125 \\
No. of primary branches/plant & $0.818^{*}$ & 0.193 & -0.085 & 0.080 & -0.136 & 0.037 & -0.048 & 0.010 \\
Seed yield/plant (g) & $0.786^{*}$ & -0.103 & 0.196 & -0.122 & 0.233 & -0.109 & -0.164 & -0.159 \\
Days to flowering & 0.130 & $0.887^{*}$ & -0.140 & 0.096 & 0.052 & -0.013 & 0.132 & 0.047 \\
Plant height (cm) & -0.019 & $0.768^{*}$ & 0.255 & 0.214 & 0.035 & -0.024 & 0.037 & 0.184 \\
Days to maturity & -0.057 & $0.642^{*}$ & 0.136 & 0.136 & 0.030 & -0.065 & 0.091 & -0.046 \\
Seeds/siliqua & 0.067 & -0.014 & $0.920^{*}$ & 0.242 & 0.048 & 0.061 & 0.117 & -0.039 \\
Main shoot length (cm) & -0.191 & -0.098 & $0.876^{*}$ & -0.317 & 0.044 & -0.061 & -0.084 & 0.035 \\
Siliqua length (cm) & 0.033 & -0.016 & 0.029 & $0.837^{*}$ & 0.042 & 0.088 & 0.102 & -0.052 \\
Siliqua density & 0.346 & 0.126 & -0.018 & $0.702^{*}$ & -0.036 & 0.145 & 0.259 & -0.102 \\
Siliqua no. main shoot & 0.255 & 0.071 & -0.088 & $0.485^{*}$ & -0.294 & 0.422 & 0.175 & -0.233 \\
No. of lobes/leaf & 0.019 & 0.029 & -0.008 & 0.024 & $0.719^{*}$ & 0.026 & 0.346 & 0.158 \\
Leaf dentations & 0.086 & 0.496 & 0.013 & 0.005 & $0.625^{*}$ & -0.041 & -0.073 & -0.311 \\
1000-seed weight (g) & -0.323 & -0.375 & 0.249 & -0.102 & $0.574^{*}$ & -0.069 & -0.185 & -0.145 \\
Oil content (\%) & -0.051 & 0.115 & 0.140 & 0.260 & -0.006 & $0.808^{*}$ & -0.123 & -0.048 \\
Leaf colour & -0.035 & 0.097 & 0.109 & 0.279 & -0.020 & -0.041 & $0.675^{*}$ & -0.051 \\
Seed colour & 0.029 & 0.113 & -0.052 & -0.049 & -0.072 & -0.035 & $0.653^{*}$ & -0.033 \\
Leaf hairness & -0.107 & 0.182 & -0.027 & -0.070 & 0.028 & -0.067 & $0.644^{*}$ & 0.052 \\
Leaf angle & -0.077 & 0.101 & 0.005 & -0.038 & 0.364 & 0.329 & -0.386 & $0.332^{*}$ \\
Siliqua angle & 0.034 & 0.165 & -0.009 & 0.027 & -0.015 & -0.026 & 0.089 & $0.854^{*}$ \\
\hline
\end{tabular}


Table 4. Clustering pattern of 96 germplasm accessions of Indian mustard.

\begin{tabular}{lll}
\hline Cluster No. & Germplasm lines & No. of lines \\
\hline CI & RC2, RC32, RC51, RC89 & 4 \\
CII & RC5, RC24 & 2 \\
CIII & RC6, RC8, RC13, RC20, RC22, RC23, RC31, RC33, RC34, RC91, RC104, RC107, & 26 \\
& RC108, RC114, RC127, RC150, RC162, RC165, RC175, RC185, RC195, RC260, & \\
& RC264, RC265, RC270, RC273 & \\
CIV & RC7, RC27, RC37, RC46, RC48, RC57, RC61, RC93, RC99, RC102, RC105, RC110, & 18 \\
& RC111, RC112, RC116, RC129, RC142, RC283 & 9 \\
CV & RC12, RC30, RC78, RC134, RC135, RC163, RC261, RC268, RC275 & \\
CVI & RC14, RC15, RC18, RC25, RC28, RC35, RC36, RC47, RC50, RC52, RC74, RC81, 25 \\
& RC85, RC86, RC106, RC118, RC148, RC152, RC154, RC164, RC166, RC171, RC174, \\
CVII & RC263, RC280 & 4 \\
CVIII & RC21, RC49, RC54, RC87 & 3 \\
CIX & RC26, RC53, RC161 & 3 \\
CX & RC29, RC38, RC77 & 2 \\
\hline
\end{tabular}

Table 5. Cluster means and general means for different characters in Indian mustard.

\begin{tabular}{|c|c|c|c|c|c|c|c|c|c|c|c|}
\hline Traits/cluster number & CI & CII & CIII & CIV & $\mathrm{CV}$ & CVI & CVII & CVIII & CIX & $\mathbf{C X}$ & $\begin{array}{l}\text { General } \\
\text { Mean }\end{array}$ \\
\hline $\begin{array}{l}\text { No. of secondary } \\
\text { branches/plant }\end{array}$ & 28.55 & 23.50 & 18.82 & 16.12 & 20.11 & 17.32 & 21.48 & 18.67 & 23.40 & 28.10 & 21.61 \\
\hline $\begin{array}{l}\text { No. of primary branch- } \\
\text { es/plant }\end{array}$ & 6.38 & 6.35 & 5.10 & 4.71 & 5.58 & 4.96 & 5.83 & 4.97 & 5.43 & 5.30 & 5.46 \\
\hline Seed yield/plant $(\mathrm{g})$ & 29.50 & 23.17 & 13.24 & 15.20 & 15.67 & 13.48 & 26.25 & 8.11 & 8.33 & 33.67 & 18.66 \\
\hline Days to flowering & 51.25 & 47.50 & 47.77 & 44.78 & 52.33 & 48.56 & 45.75 & 55.00 & 44.67 & 46.00 & 48.36 \\
\hline Plant height (cm) & 224.20 & 203.95 & 215.10 & 195.10 & 245.52 & 228.12 & 209.63 & 244.40 & 178.20 & 199.10 & 214.33 \\
\hline Days to maturity & 150.75 & 144.50 & 145.08 & 145.28 & 146.44 & 146.96 & 147.25 & 150.33 & 144.00 & 144.00 & 146.46 \\
\hline Seeds/siliqua & 13.05 & 14.10 & 12.58 & 12.87 & 12.08 & 11.85 & 13.40 & 13.63 & 12.40 & 10.80 & 12.68 \\
\hline Main shoot length $(\mathrm{cm})$ & 73.98 & 55.95 & 77.02 & 86.63 & 77.42 & 94.52 & 95.58 & 56.30 & 59.33 & 82.95 & 75.97 \\
\hline Siliqua length $(\mathrm{cm})$ & 3.30 & 3.50 & 3.28 & 3.51 & 3.34 & 3.37 & 3.63 & 3.27 & 3.40 & 2.85 & 3.35 \\
\hline Siliqua density & 0.74 & 0.88 & 0.70 & 0.66 & 0.73 & 0.67 & 0.70 & 0.77 & 0.65 & 0.76 & 0.73 \\
\hline Siliqua no. main shoot & 54.58 & 49.45 & 54.05 & 56.33 & 56.60 & 63.56 & 66.65 & 43.30 & 38.63 & 62.80 & 54.59 \\
\hline No. of lobes/leaf & 5.50 & 4.00 & 5.35 & 5.33 & 5.44 & 4.64 & 5.25 & 4.00 & 4.00 & 4.00 & 4.75 \\
\hline Leaf dentations & 4.50 & 3.00 & 4.46 & 4.78 & 2.78 & 4.28 & 5.50 & 3.00 & 4.33 & 4.00 & 4.06 \\
\hline 1000-seed weight $(\mathrm{g})$ & 3.20 & 2.63 & 3.14 & 3.84 & 3.01 & 3.42 & 3.55 & 2.64 & 3.03 & 3.15 & 3.16 \\
\hline Oil content $(\%)$ & 39.80 & 39.50 & 40.25 & 39.73 & 39.59 & 40.35 & 40.55 & 40.23 & 39.10 & 39.30 & 39.84 \\
\hline Leaf colour & 1.75 & 2.00 & 1.96 & 1.94 & 2.33 & 1.92 & 2.25 & 2.33 & 1.67 & 2.00 & 2.02 \\
\hline Seed colour & 3.00 & 3.00 & 2.77 & 3.11 & 3.33 & 2.88 & 3.50 & 3.00 & 3.67 & 3.00 & 3.13 \\
\hline Leaf hairness & 2.50 & 2.00 & 2.77 & 3.22 & 3.00 & 3.00 & 2.50 & 7.00 & 3.00 & 3.00 & 3.20 \\
\hline Leaf angle & 5.00 & 4.00 & 5.08 & 5.22 & 5.67 & 5.08 & 5.00 & 5.00 & 5.00 & 6.00 & 5.11 \\
\hline Siliqua angle & 4.50 & 5.00 & 4.77 & 4.78 & 5.67 & 5.08 & 4.00 & 5.67 & 5.00 & 6.00 & 5.05 \\
\hline
\end{tabular}

Table 6. Inter-and intra-cluster distances in Indian mustard accessions

\begin{tabular}{|c|c|c|c|c|c|c|c|c|c|c|}
\hline Cluster & CI & CII & CIII & CIV & $\mathrm{CV}$ & CVI & CVII & CVIII & CIX & $\mathbf{C X}$ \\
\hline $\mathrm{CI}$ & 41.00 & & & & & & & & & \\
\hline CII & 80.94 & 30.36 & & & & & & & & \\
\hline CIII & 75.71 & 78.27 & 52.17 & & & & & & & \\
\hline CIV & 99.33 & 88.93 & 68.58 & 48.52 & & & & & & \\
\hline $\mathrm{C} \mathrm{V}$ & 81.22 & 109.93 & 80.18 & 104.28 & 49.05 & & & & & \\
\hline C VI & 89.33 & 115.75 & 71.02 & 82.51 & 75.94 & 47.04 & & & & \\
\hline CVII & 83.32 & 97.75 & 77.33 & 74.41 & 106.63 & 71.18 & 34.00 & & & \\
\hline C VIII & 106.99 & 102.80 & 103.19 & 138.72 & 84.11 & 111.66 & 154.78 & 38.88 & & \\
\hline C IX & 129.16 & 81.26 & 100.03 & 94.5 & 139.93 & 141.89 & 138.51 & 114.89 & 24.12 & \\
\hline $\mathrm{CX}$ & 96.41 & 88.85 & 82.43 & 77.26 & 107.09 & 94.97 & 66.88 & 157.77 & 114.41 & 26.20 \\
\hline
\end{tabular}

accessions i.e.26 were grouped in Cluster III (CIII) followed by 25 lines in cluster VI (CVI) whereas, only two accessions each were grouped in clusters CII and CX. The clusters I, IV, V, VII, VIII and IX comprised 4, 18, 9, 4, 3 and 3 lines, respectively. Similar results were obtained by Doddabhimappa et al. (2010), Singh (2012) and Neeru et al. (2015).
Using the principal factor scores (PF scores), graph was plotted to represent the position of genotypes on $\mathrm{X}$ and $\mathrm{Y}$-axis by taking two most important factors i.e. yield factor and oil factor and to chalk out the breeding plan for further improvement by identifying superior parents for hybridization/ crossing programme. In Fig. 1, all the accessions were plotted for PF1 (seed yield 
and its important components) and PF6 (oil content). Based on PF scores and cluster mean values (Table 5) the accessions viz.RC2, RC32 and RC51 (cluster I), RC95 and RC96 (cluster X) were found superior for seed yield/plant and yield related factors like primary and secondary branches/plant; while the accessions RC34, RC185 and RC195 (cluster III) and RC53 (cluster VIII) were found superior for oil content. From the foregoing discussion it can decisively be concluded that these accessions can be used as parents in hybridization programme for evolving Indian mustard varieties with high seed yield and oil content as well or for obtaining transgressive segregants superior for these traits of superiority in the segregating generations. Also, based on the PF scores and cluster mean values, the germplasm lines RC21, RC49 and RC54 were found superior for both yield and oil content. Similarly, Alemayehu and Becker (2002) found that both principal component and cluster analyses disclosed complex relationships among the Ethiopian mustard (Brassica carinata A. Braun) accessions and characters.

The perusal of the data in Table 5, reveals that CI comprised germplasm accessions with more number of primary branches/plant, secondary branches/plant and seed yield. Similarly, genotypes grouped in CII had high siliqua density, semi-appressed siliqua angle and more seeds/siliqua. Cluster III was characterized with the accessions having high oil content and medium green foliage colour. Accessions of CIV had bold seeds and medium earliness in days to flowering. Lines grouped in $\mathrm{CV}$ were characterized with lesser leaf dentations, higher number of lobes/leaf and open type of leaf angle. High siliqua number on main shoot and high oil content were observed in the member accessions of CVI whereas, CVII was characterized with high siliqua number on main shoot, more siliqua length, high oil content and high seed yield/plant. The accessions grouped in CVIII were characterized with more leaf hairiness and high oil content. Cluster IX had lines with less plant height, medium earliness in flowering, semi-appressed siliqua angle and seed colour varied between red-brown to brown. Cluster X was having only two accessions which recorded the highest seed yield/plant.

As hybridization among diverse parents is likely to produce heterotic hybrids and desirable transgressive segregants in further generations, grouping germplasm lines in different clusters gives an opportunity for selecting them to serve the objectives in developing genotypes with specific characters. To assess the diversity inter and intra-cluster distances were calculated which are presented in Table 6 . Inter-cluster distance was maximum between clusters VIII and X (157.77) followed by between CVII and CVIII (154.78) and CVI and CIX (141.89), whereas, the minimum inter-cluster distance was observed between CVII and CX (66.88) followed by CIII and CIV (68.58). The crosses between the germplasm lines belonging to distantly located clusters are likely to produce good transgressive segregants and germplasm lines with better mean values can be selected among all the genotypes to suit the breeding programme. Maximum intra-cluster distance was observed in the cluster III (52.17) followed by in CV (49.05) and minimum in the CIX (24.12). Similarly, Pandey et al. (2013) evaluated 45 Indian mustard genotypes for the extent of diversity present and the maximum inter-cluster distance was found between clusters II and III indicating high genetic divergence among genotypes of these groups. Based on the results of the present study, it is recommended to use the diverse accessions RC2, RC32 and RC51 (cluster I), $\mathrm{RC} 95$ and RC96 (cluster X); and RC34, RC185 and RC195 (cluster III) and RC53 (cluster VIII) as one of the parents for improving seed yield, its important components along with oil content. These were the accessions which also got plotted on the better ends of scatter plot based on PF scores and hence, the results of cluster analysis and principal factor analysis confirmed each other.

\section{Conclusion}

On the basis of present study, it can be concluded that the Indian mustard germplasm accessions having high seed yield/plant and yield contributing component traits viz., RC2 (33.3 g), RC32 (30.7 g), RC51 (29.3 g), RC95 $(35.0 \mathrm{~g})$ and RC96 (32.3 g); and the accessions with superior oil content viz. RC34 (41.1\%), RC53 (42.2\%), RC185 (40.0\%) and RC195 (40.7\%) can be utilized in trait combination breeding programmes for evolving mustard varieties with high seed yield and oil content.

\section{REFERENCES}

Alemayehu, N. and Becker, H. (2002). Genotypic diversity and patterns of variation in a germplasm material of Ethiopian mustard (Brassica carinata A. Braun) Genet. Resources Crop Evo. 49: 573-582.

Anonymous (2015). "Agricultural statistics at a glance." Directorate of Economics and Statistics, Department of Agriculture, Co-operative and Farmers Welfare. pp 122.

Avtar, R., Singh, D., Thakral, N.K., Singh, A., Sangwan, O., Rani, B. and Kumari, N. (2014). Multivariate analysis for evaluation and classification of toria germplasm accessions. Res. Crops 15(1): 129-134.

Doddabhimappa, R., Gangapur, B., Prakash, G. and Hiremath, C. P. (2010). Genetic Diversity analysis of Indian mustard (Brassica juncea L.) Electro. J. Plant Breed. 1(4): 407-413.

Nagaharu U. (1935). Genome analysis in brassica with special reference to experimental formation of B. napus and peculiar mode of fertilization. Jap. J. Bot. 7: 389452.

Neeru, Thakral, N. K., Avtar R., and Singh A. (2015). Evaluation and classification of Indian mustard (Brassica juncea L.) genotypes using principal component analysis. J. Oilseed Brassica 6(1), 167-174. 
Pandey, R., Kumar, B. and Kumar, M. (2013). Genetic divergence for quantitative traits in Indian mustard (Brassica juncea L. Czern \& Coss). American-Eurasian J. Agric. \& Environ. Sci. 13 (3): 348-351, 2013.

Romesburg, H.C. (1984). Cluster Analysis for Researchers. Krieger Publishing Co., Malabar, Florida.

Singh, B. (2012). Genetic divergence in elite genotypes of Indian mustard (Brassica juncea L.). M.Sc. Thesis; CCS HAU, Hisar.

Singh, K. H., Shakya, R. and Mahawar, R. K. (2014). Genetic diversity and patterns of variation among Indian mustard (Brassica juncea (L.) Czern \& Coss.) varieties. SABRAO J. Breed. Genet. 46(2): 329-339.
Singh K. H., Shakya, R., Thakur, A. K., Chauhan, D. K. and Chauhan, J. S. (2013). Genetic diversity in Indian mustard [Brassica juncea (L.) Czernj \& Cosson] as revealed by agronomic traits and RAPD markers. Natl. Acad. Sci. Lett. 36: 419-427.

Zada, M., Zakir, N., Rabbani, M., Shinwari, A. and Khan, Z. (2013). Assessment of genetic diversity in Ethiopian mustard (Brassica carinata A. Brun) germplasm using multivariate techniques. Pak. J. Bot. 45(SI): 583-593.

Zaman, M. A., Khatun, M. T., Ullah, M. Z., Moniruzzamn, M. and Rahman, M. Z. (2010). Multivariate analysis of divergence in advanced lines of mustard (Brassica spp) Bangladesh Journal of Plant Breeding Genet. 23(2):29-34. 